\title{
Structural Condition Assessment of the Herringbone Middle Pylon of the Taizhou Bridge Using SHM Strain Data
}

\author{
Huaping Ding, Qinghong Shen, and Sidan Du \\ School of Electronic Science and Engineering, Nanjing University, Nanjing 210023, China \\ Correspondence should be addressed to Sidan Du; coff128@nju.edu.cn
}

Received 6 May 2017; Revised 16 July 2017; Accepted 26 July 2017; Published 7 September 2017

Academic Editor: Mosbeh Kaloop

Copyright (C) 2017 Huaping Ding et al. This is an open access article distributed under the Creative Commons Attribution License, which permits unrestricted use, distribution, and reproduction in any medium, provided the original work is properly cited.

\begin{abstract}
Taizhou Bridge is the world's first kilometer-scale three-pylon suspension bridge. To minimize the impacts on navigation, a longitudinal herringbone steel pylon was adopted in the middle of the bridge without additional piers. This structure is unique, and little research has focused on its structural condition assessment. In this paper, eighty fiber Bragg grating strain sensors were deployed along the height of the steel tower to collect strain data about the key components and to monitor the pylon's structural condition. Because temperature-induced strain contributes little to the stress in the pylon, the empirical mode decomposition method was proposed to remove the noise and the temperature-induced strain, leaving the dynamic strain response. The frequency characteristics were obtained from both the dynamic strain and the raw strain, and they show good agreement. A statistical analysis was adopted assuming that the extracted dynamic stress peaks and valleys were normally distributed. The expected maximum values from the statistical analysis were compared with the measured maximum values at different heights, and they agree well with each other. The maximum compression and tension of the key segments of the middle tower exhibited considerable redundancy, which indicates that the middle pylon is in good condition.
\end{abstract}

\section{Introduction}

Large-scale suspension bridges with main spans exceeding $1 \mathrm{~km}$ have been an increasing focus of research in the bridge engineering community and have been implemented worldwide due to their longer main spans, lower construction costs, and facilitation of navigation. Typical large-scale suspension bridge projects include the Golden Gate Bridge in the USA [1], the Humber Bridge in the UK [2], the Tsing Ma Bridge in Hong Kong [3], the Akashi Kaikyo Bridge in Japan [4], the Great Belt Bridge in Denmark [5], the Bosphorus Bridge in Turkey [6], and the Runyang Bridge [7] and Jiangyin Bridge [8] over the Yangtze River in China. After the completion of construction, the majority of these large-scale bridges were instrumented with long-term structural health monitoring (SHM) systems for structural condition assessment. For example, a sophisticated bridge safety monitoring system, known as the wind and structural health monitoring system (WASHMS), was installed in 1997 to monitor the durability and reliability of the Tsing Ma Bridge, which has a $1,377 \mathrm{~m}$ long main span [9]. The project consists of approximately
280 sensors, including strain sensors, accelerometers, and displacement transducers. All essential information collected from the sensors is transmitted to the data acquisition system for further structural safety assessment.

In an SHM system, strain data can provide information about the actual local behavior of the structural components under operational conditions. Based on the analysis of strain data, the stress and fatigue life of the structures can be obtained. As a result, many researchers have proposed several methods to analyze strain data for structural condition assessment. For example, Catbas et al. [10] proposed a parametric-based approach to model temperature-induced strain responses using a linear-regression model. They demonstrated that the temperature-induced strain response was not easy to conceptualize and model using conventional parametric methods. Ni et al. [11, 12] proposed a waveletbased approach to assess the structural condition of the Tsing Ma Bridge deck using in-service SHM monitoring strain data. Wang et al. [13] used the wavelet packet decomposition method to extract the strain induced by the static temperature for a correlation analysis based on the SHM system 
installed on the Dashengguan Yangtze Bridge. Deng et al. [14] studied the fatigue performance of welded details using measured strain data of the Runyang Suspension Bridge. They demonstrated that the temperature has a negligible effect on the stress range spectrum and used the wavelet transform decomposition technique to remove the effect of temperature. These analyses are all based on the wavelet transform. The fundamental procedure of wavelet-based methods is to select suitable basis functions for the signals. However, the selection of basis functions is not always precise and thus may affect the accuracy of the results.

This study proposes an approach for structural condition assessment of a bridge tower using in-service SHM strain data and applies it to Taizhou Bridge, which is the world's first three-pylon kilometer-scale suspension bridge and it crosses the Yangtze River in Jiangsu, China [15, 16]. The bridge is unique because of its superlong spans and its longitudinal inverted Y-shaped steel tower. The approach consists of three procedures: (1) based on the fact that the raw signal is a combination of strains due to wind, temperature, traffic loads, and noise and that the temperature-induced strain is considerable but contributes little to the stress [17], an empirical mode decomposition (EMD) method is proposed to remove the noise and temperature-induced strain and extract the dynamic strain. The proposed EMD method is more flexible than traditional parametric methods, which are limited by stationarity and the Gaussian noise assumption. Furthermore, in contrast to the wavelet transform, the proposed method is adaptive without any manual selection and is suitable for nonstationary and nonlinear processes [18]. (2) Based on the dynamic strain, the frequency characteristics of the tower are obtained and compared to the raw data, and the peak stresses are derived and then adopted to simulate the distribution. (3) The statistical characteristics of the peak stresses at different heights are explored based on the simulation results.

The paper provides a new perspective on Taizhou Bridge for academic researchers and project engineers and reveals the stress characteristics of this unique structure, which has seldom been adopted and studied. The whole analysis process can be used for structural condition assessment of this type of bridge, and the results provide scientific references for inspection and maintenance of the bridge.

\section{Taizhou Bridge and Its Central Pylon}

The Taizhou Yangtze River Highway Bridge, which links Taizhou City and Zhenjiang City, is the sixth cable-supported large-scale bridge across the Yangtze River in Jiangsu Province, China (after the Jiangyin Bridge in 1999, the 2nd Nanjing Bridge in 2001, the Runyang Bridge in 2005, the 3rd Nanjing Bridge in 2005, and the Sutong Bridge in 2008). Figure 1 shows the location of the Taizhou Bridge.

In contrast to the other bridges, the Taizhou Bridge, which was opened to traffic in November 2012 and carries a two-way, six-lane highway, is the first kilometer-scale threepylon suspension bridge in the world. It includes an inverted Y-shaped middle tower and two continuous $1,080 \mathrm{~m}$ long

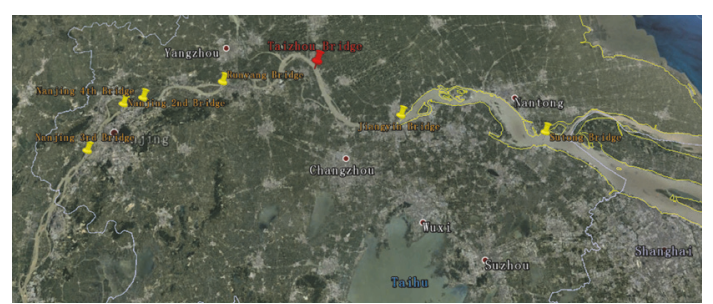

Figure 1: Location of the Taizhou Bridge.

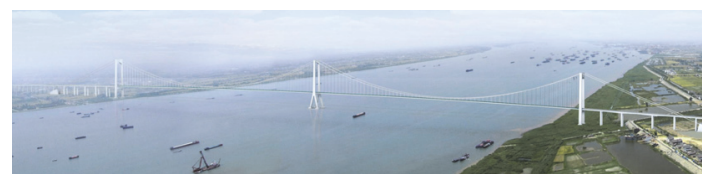

Figure 2: Overview of the Taizhou Bridge.

main spans. Figure 2 shows an overview of the Taizhou Bridge.

The unique bridge type, which uses fewer piers to support the entire structure, was proposed and implemented considering the environmental impacts, flood discharge impact, facilitation of navigation, and safety of the structure. The three foundations of the pylons are erected on a riverbed with a W-shaped cross-section. The central pylon, which is founded on a $58 \mathrm{~m}$ by $48 \mathrm{~m}$ caisson, has a longitudinal inverted Y-shaped structure, is constructed of steel and is $200 \mathrm{~m}$ high. Due to the stiffness of the middle pylon, the stresses in the main cables and anchor blocks are lower than those in a traditional two-pylon bridge. The force transfers and dynamic loads lead to complex stresses in the steel middle pylon. The side pylons are $180 \mathrm{~m}$ tall concrete frame structures that are supported by 46 friction poles. Figure 3 shows the layout of the middle pylon.

The steel tower column is divided into 21 segments, which are denoted D0 to D20, as shown in Figure 3. Because they are subjected to different forces, the segments below a height of $135.04 \mathrm{~m}$ (D0-D12) use Q420QD steel (elastic modulus $E=$ $2.1 e 11 \mathrm{~Pa}$, yield strength $=420 \mathrm{MPa}$ ), whereas the remainder, including segments D13-D20 and both beams, use Q370QD steel (modulus of elasticity $E=2.1 e 11 \mathrm{~Pa}$, yield strength $=370 \mathrm{MPa}$ ). M30 friction-type high strength bolts are used to connect the neighboring segments. Segment D0 is at the bottom, and D1-D3 are on the leg of the tower. Segment D4 has two legs and is the most complex structure; it joins D3 (two segments), D5, and the lower cross-beam. All of the other segments, starting from D6, are in a line, and D13 is the point that connects the segments with two types of steel.

Segment D5 has the maximum length of $15 \mathrm{~m}$ and is also the heaviest segment with a weight of $495,000 \mathrm{~kg}$. Because of the unavoidable deviations during manufacturing of the segments and the limitations of the measurement instrumentation, four adjusting components (connectors) located between D0-D1, D3-D4, D5-D6, and D15-16 were used to adjust the segments during installation to achieve sufficient straightness of the tower. 


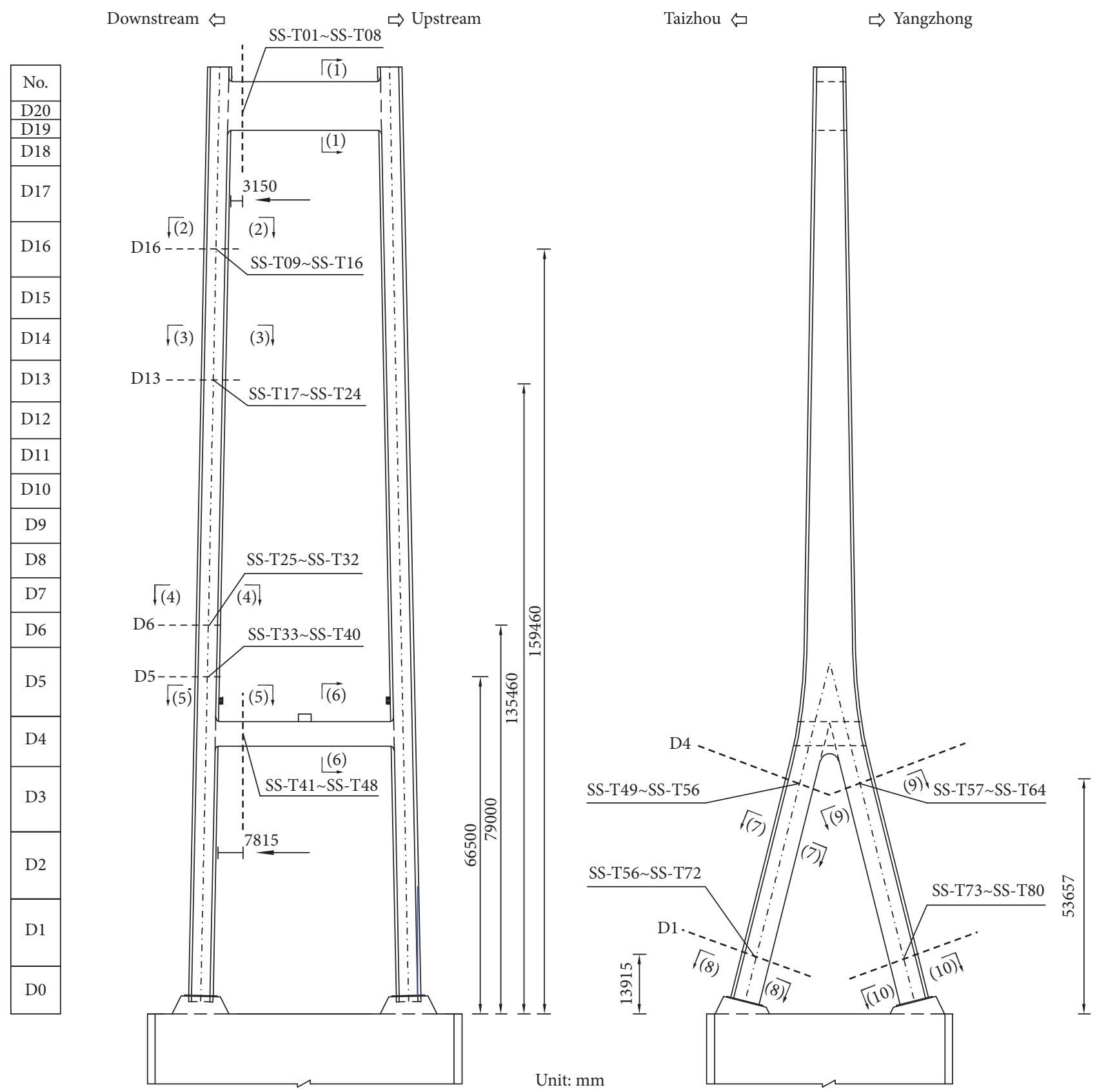

Figure 3: Layout of the middle pylon.

\section{Installation of the ESHM System and SSM System}

After construction of the bridge, a long-term environmental and structural health monitoring system (ESHMS) was instrumented to monitor the bridge's environmental and structural condition and assess the bridge's structural health performance. As shown in Figure 4, the ESHMS consists of two subsystems: the environmental monitoring system (EMS) and the structural health monitoring system (SHMS), which includes the structural alignment monitoring system (SAMS), structural vibration monitoring system (SVMS), structural strain monitoring system (SSMS), and structural temperature monitoring system (STMS).

A total of 275 sensors (summarized in Table 1), including three-dimensional ultrasonic anemometers, thermohygrometers, global positioning systems, acceleration sensors, strain sensors, and temperature sensors, were installed to collect different types of data for the ESHM system.

Figure 5 shows the installation positions of all 275 sensors, which were deployed on the main cables, decks, pylons, and suspender cables. As part of the bridge, the middle pylon makes the bridge unique in terms of its structural stress and structural response. On the downstream side of the 

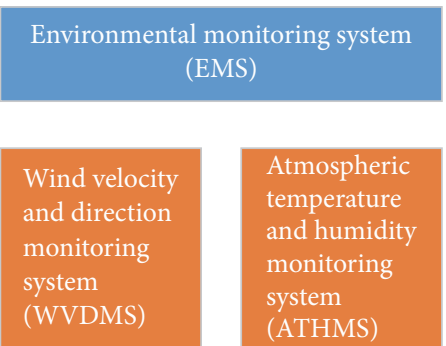

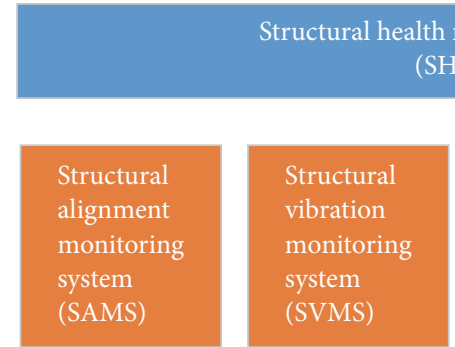

(SHMS)

FIGURE 4: ESHM architecture.

TABLE 1: Sensor types and quantities.

\begin{tabular}{|c|c|c|c|}
\hline Number & \multicolumn{2}{|c|}{ Sensor type } & Quantity \\
\hline 1 & \multicolumn{2}{|c|}{ Three-dimensional ultrasonic anemometer } & 1 \\
\hline 2 & \multicolumn{2}{|c|}{ Thermohygrometer } & 1 \\
\hline \multirow{2}{*}{3} & \multirow{2}{*}{ Global positioning system } & Reference station & 1 \\
\hline & & Receiving station & 11 \\
\hline 4 & \multicolumn{2}{|c|}{ Acceleration sensor } & 51 \\
\hline 5 & \multicolumn{2}{|c|}{ Strain sensor } & 168 \\
\hline 6 & \multicolumn{2}{|c|}{ Temperature sensor } & 42 \\
\hline \multicolumn{3}{|c|}{ Total } & 275 \\
\hline
\end{tabular}

TABLE 2: Characteristics of the FBG1550-SS100 strain sensors.

\begin{tabular}{lcc}
\hline Number & Characteristic & Value \\
\hline 1 & Measurement range & $\pm 1500 \mu \varepsilon$ \\
2 & Resolution & $0.1 \mu \varepsilon$ \\
3 & Accuracy & $\pm 0.01 \% \mathrm{~F} . S$ \\
4 & Sensitivity & $1.18 \sim 1.22 \mathrm{pm} / \mu \varepsilon$ \\
5 & Working temperature range & $-30^{\circ} \mathrm{C}-70^{\circ} \mathrm{C}$ \\
6 & Wavelength range & $1525 \mathrm{~nm}-1565 \mathrm{~nm}$ \\
\hline
\end{tabular}

middle pylon, 80 strain sensors were installed in 10 sections to determine its structural characteristics.

Figure 6 shows the detailed installation positions of the 80 strain sensors in segments D1 (8-8), D4 (7-7), D5 (5-5), D6 (44), D13 (3-3), and D16 (2-2), which have heights of $13.915 \mathrm{~m}$, $53.657 \mathrm{~m}, 66.5 \mathrm{~m}, 79 \mathrm{~m}, 135.46 \mathrm{~m}$, and $159.46 \mathrm{~m}$, respectively, and in the upper cross-beam (1-1) and lower cross-beam (66) of the tower near the downstream side. Every section has 8 strain sensors. The sensors on the upstream Yangzhong side were chosen for the analysis of the change in height. These sensors are marked in red in the figure. The characteristics of the strain sensors are listed in Table 2.

\section{Data Processing Methods}

4.1. Extraction of the Dynamic Strain from the Original Signal. The strain data of the bridge were acquired at a sampling rate of $20 \mathrm{~Hz}$ from the ESHMS. They are typically nonstationary signals that include a combination of wind-, temperature-, and traffic-induced strains and noise. The temperatureinduced strain is considerable but contributes little to the stress because most of it is released by the free movement of the pylon at the four adjusting connectors $[11,12]$. Because the EMD method is adaptive and suitable for nonstationary and nonlinear processes, it was used to remove the temperatureinduced strain and noise. By decomposing a signal into intrinsic mode functions (IMFs), the EMD method reveals multiple causes mixed in the signal without any manual selection. The process of extracting an IMF is as follows: (1) let $x(t)$ be the original signal and find all of the local maxima and minima of $x(t)$; (2) connect all of the local maxima and minima as the upper and lower envelopes by a cubic spline; (3) assume $u_{1}(t)$ is the mean value of both envelopes and compute the difference between the original signal and the mean value

$$
h_{1}(t)=x(t)-u_{1}(t)
$$

(4) determine whether $h_{1}(t)$ satisfies the definition of an IMF. If not, repeat the previous process by taking $h_{1}(t)$ as a new signal until $h_{1}(t)$ meets the requirement to be an IMF; (5) the final result becomes the first IMF component, which is designated $c_{1}(t)$, and the residue $r_{1}(t)$ is obtained as

$$
r_{1}(t)=x(t)-c_{1}(t) .
$$

The sifting process can be repeated by treating each subsequent residue as the new signal and stops if the final residue $r_{n}(t)$ is smaller than a predetermined value or 


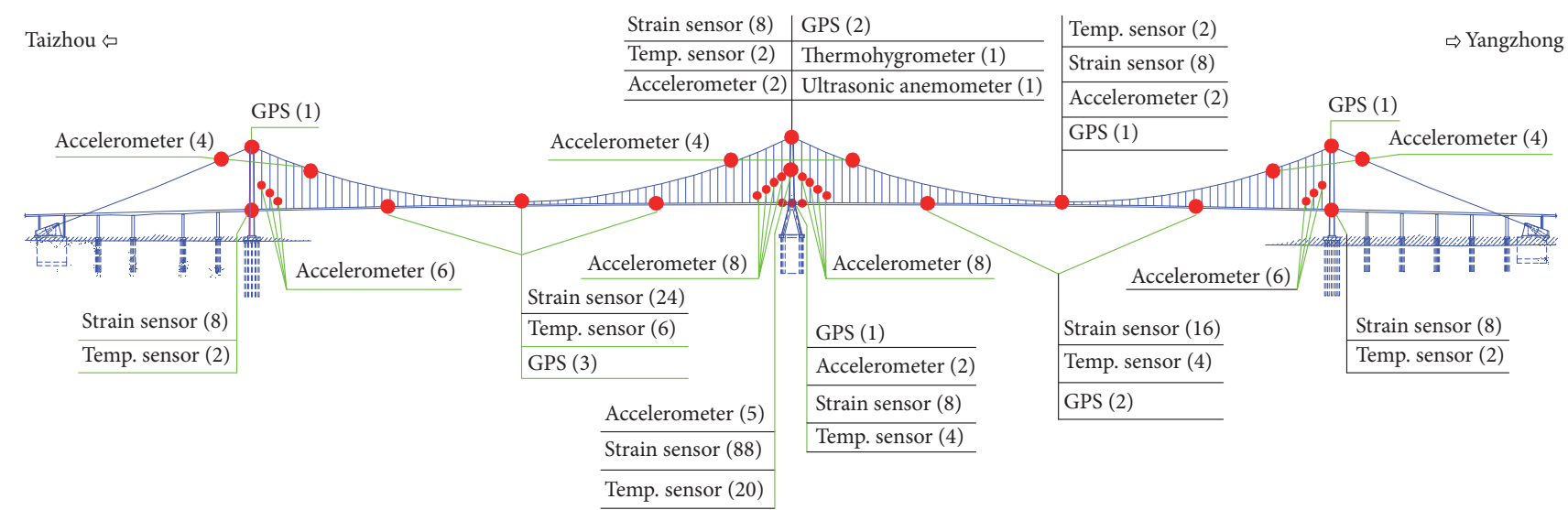

FIGURE 5: Installation of the ESHM sensors (bridge elevation).

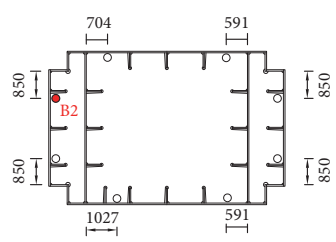

Yangzhong $\Leftrightarrow \quad \Rightarrow$ Taizhou

$2-2$

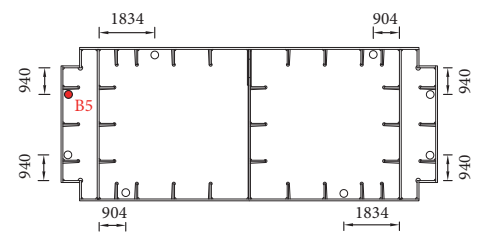

Yangzhong o

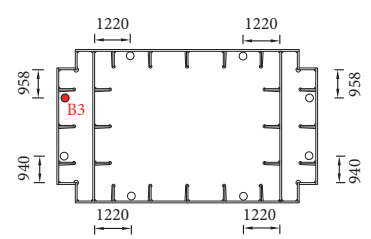

طangzhong

$\Rightarrow$ Taizhou

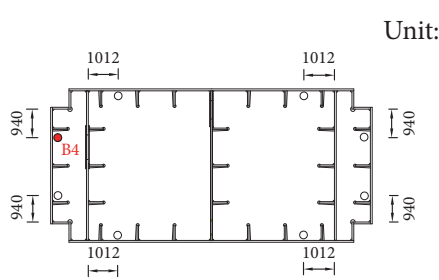

$\Rightarrow$ Taizhou

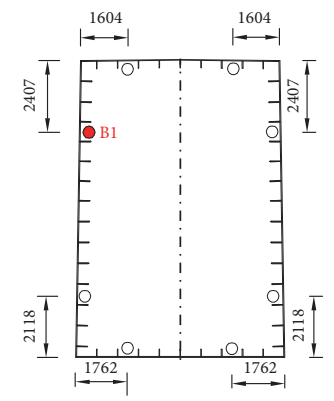

Yangzhong $\diamond \quad \Rightarrow$ Taizhou $1-1$
$\Rightarrow$ Taizhou 5-5
$3-3$

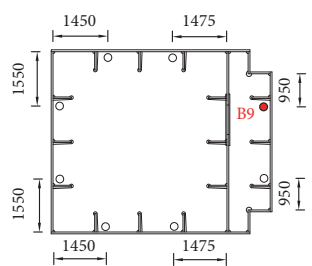

$\Rightarrow$ Yangzhong

9-9

(a)
Taizhou $\bullet$

Yangzhong s

$4-4$

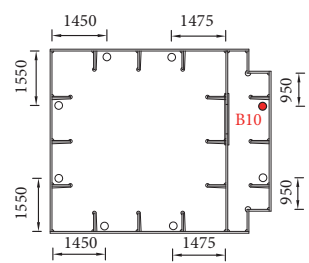

Taizhou s

10-10

Unit: $\mathrm{mm}$

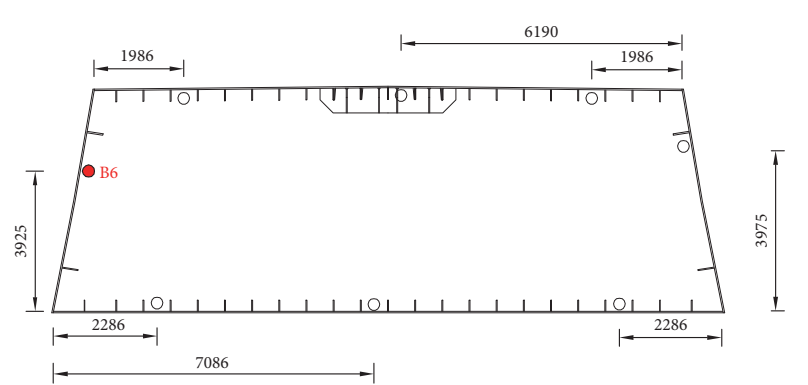

Yangzhong ط

$\Rightarrow$ Taizhou

(b)

FiguRE 6: Distribution of the 80 strain sensors in the middle pylon: (a) in the segments; (b) in the beams. 


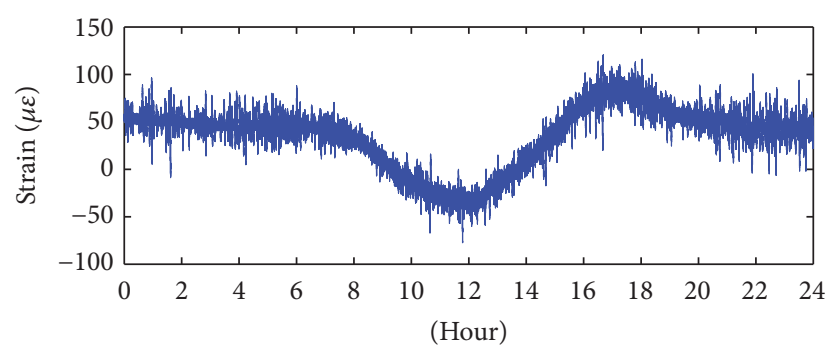

FIgURE 7: The original strain of B3 on 2013-05-02.

becomes a monotonic function. Thus, the original signal can be decomposed into a collection of IMFs plus the final residue, which is expressed as

$$
x(t)=\sum_{i=1}^{\mathbf{n}} c_{i}(t)+r_{n}(t) .
$$

To demonstrate the EMD method, Figure 7 shows the original signal of B3 on 2013-05-02, which includes a combination of temperature-induced strain, dynamic strain, and noise. The temperature-induced strain is large and has a clear trend in the time history.

After decomposition using the EMD method, the 24 IMFs and residues and their power spectrum densities (PSDs) are obtained as shown in Figure 8. The dynamic strains are extracted by removing the trend components and noise, which are identified through the characteristics of the high frequency response.

Figure 9 shows the temperature-induced strain and the dynamic strain after applying the EMD method to decompose the complex strain data of B3 on 2013-05-02. In the figure, negative values indicate tensile strains, and positive values indicate compressive strains. The temperature-induced strain ranges from approximately $-50 \mu \varepsilon$ to $100 \mu \varepsilon$; however, the dynamic strain is relatively small with an approximate range of $-60 \mu \varepsilon$ to $60 \mu \varepsilon$. The alternating compressive and tensile strains are excited because both sides of the middle pylon are under asymmetric loads.

4.2. Frequency Response of the Middle Tower. The natural frequency is one of the most important characteristics that can reveal a structure's health status. To explore the frequency characteristics of the middle tower, the PSDs of the measured data at different heights are calculated as shown in Figure 10. The vibration modes are clearly the same at different heights, although some modes are not significant or are not excited at heights of $13.915 \mathrm{~m}$ and $159.46 \mathrm{~m}$. The first, second, third, and fourth obvious peaks are determined to be 0.07813 , $0.1172,0.166$, and $0.2197 \mathrm{~Hz}$, respectively. These modes are identified as the first lateral mode, second vertical mode, fifth vertical mode, and third lateral mode, respectively, as reported in $[19,20]$. The relative errors between the measured natural frequencies and the calculated frequencies $(0.07742$, $0.11776,0.16898$, and $0.22168 \mathrm{~Hz}$, resp.) are $0.92 \%,-0.48 \%$, $-1.76 \%$, and $-0.89 \%$, respectively. These results indicate that the bridge is in a stable condition.
Figure 11 shows the frequency responses from both the original signal and the dynamic strain after removing the temperature effect and noise using the EMD method. The two spectrums are nearly identical. Therefore, the temperature variation and noise have little effect on the frequency spectrums, and they can be ignored.

4.3. Extraction of the Peaks and Valleys from the Dynamic Strain. The stresses are obtained by multiplying the strains by the modulus of elasticity of the steel. The stresses and their peaks are random variables. Because large stresses greatly contribute to fatigue failure, a statistical analysis of the peak stresses is vital for structural condition assessments. Thus, small stresses contribute little to structural fatigue and should be neglected in the analysis. Figure 12 shows the stress peaks and valleys recorded on 2013-05-02 from the processed stresses of $B 3$ induced by the live loads, which were determined using the MATLAB function of finding local peaks in data.

\subsection{Calculation and Simulation of the Stress Distribution.} The MATLAB functions for computing the kernel density and estimating a distribution were used to calculate the distribution of the stress peaks of B3 on 2013-05-02. The distribution of strain peaks exhibits a bell-shaped curve, as shown in Figure 13. Hence, a Gaussian distribution is adopted to analyze the stress peaks and valleys.

Figure 14 shows how well the Gaussian curve fits the distribution of the stress peaks. The model function is determined by the MATLAB curve fitting tool, and the coefficients are for the $95 \%$ confidence bounds.

$$
\begin{aligned}
f(x)= & 0.1783 * \exp \left(-\left(\frac{x-2.102}{1.886}\right)^{2}\right)+0.06567 \\
& * \exp \left(-\left(\frac{x-3.506}{3.428}\right)^{2}\right)
\end{aligned}
$$

The $R$-squared statistic measures how successful the fit is in explaining the variation of the data. $R$-squared values range from 0 to 1 , and a value close to 1 indicates a better fit. The $R$ squared value of the data is 0.9981 , which indicates a perfect fit.

\section{Results and Discussion}

5.1. Stress Analysis along the Height. The statistical properties, including the mean values, standard deviations (SDs), and the measured and expected maximum stresses, are listed in Table 3. The expected maximum stresses were calculated by adding the triple standard deviation to the mean value $(\mu+3 \sigma)$. Table 3 illustrates that the maximum expected compressive stresses are generally lower than $12 \mathrm{MPa}$ and have a maximum value of $11.8275 \mathrm{MPa}$ at a height of $66.5 \mathrm{~m}$; the maximum expected tension is $-13.1245 \mathrm{MPa}$ and occurs at the same height, which reveals that the segments have considerable redundancy. The highest mean compressive stress is $4.70475 \mathrm{MPa}$ at a height of $66.5 \mathrm{~m}$, which is 3.80 times and 2.32 times higher than those at heights of $13.915 \mathrm{~m}$ 

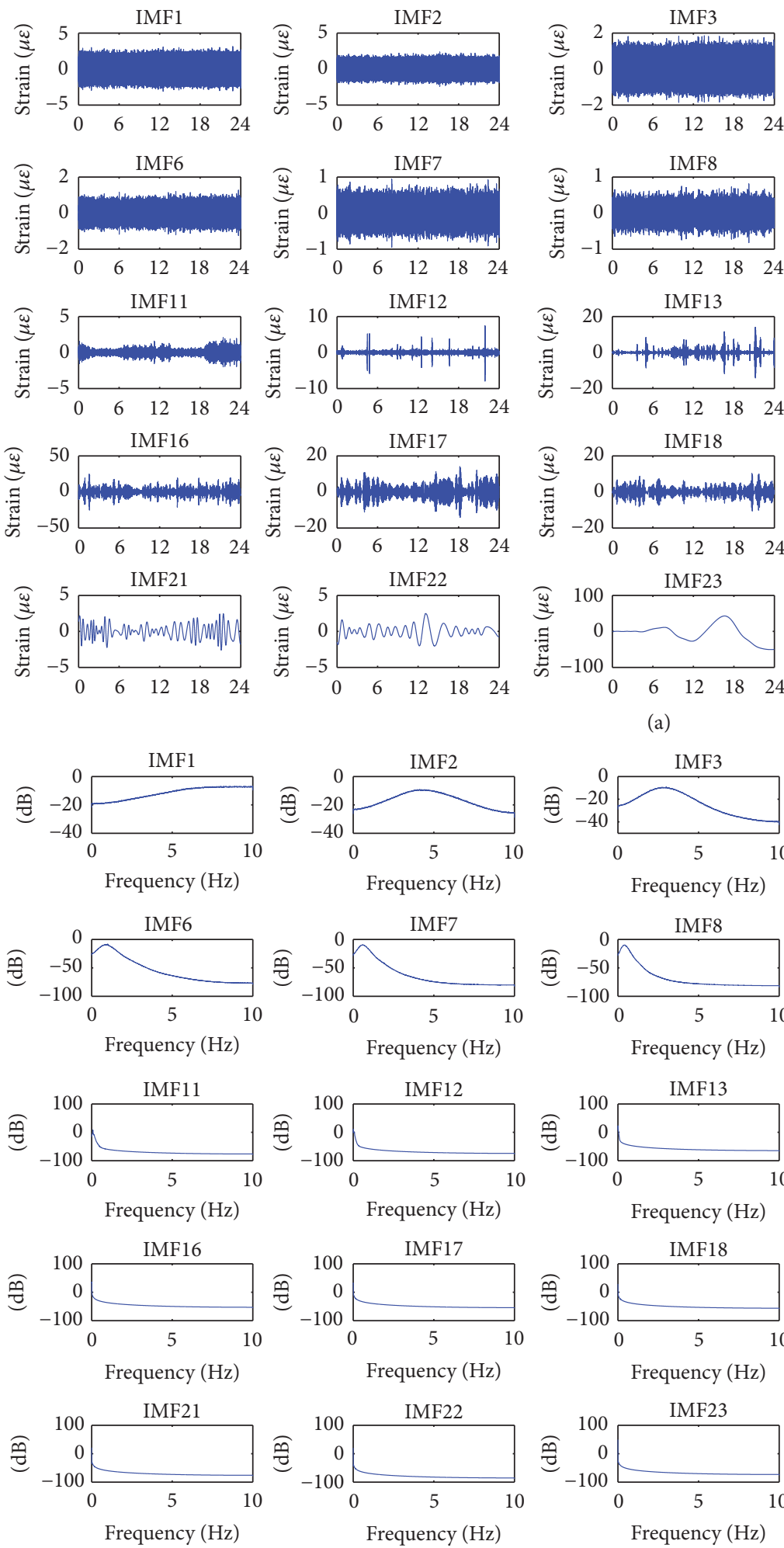
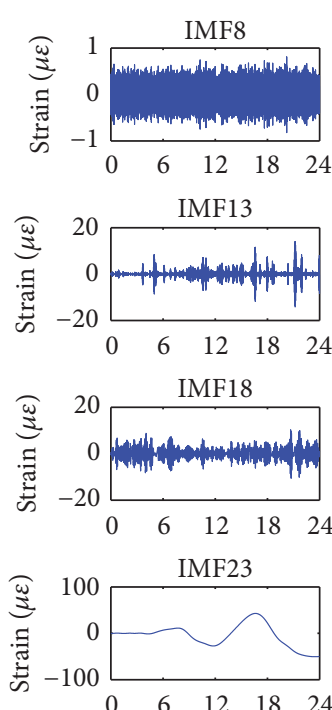

(a)
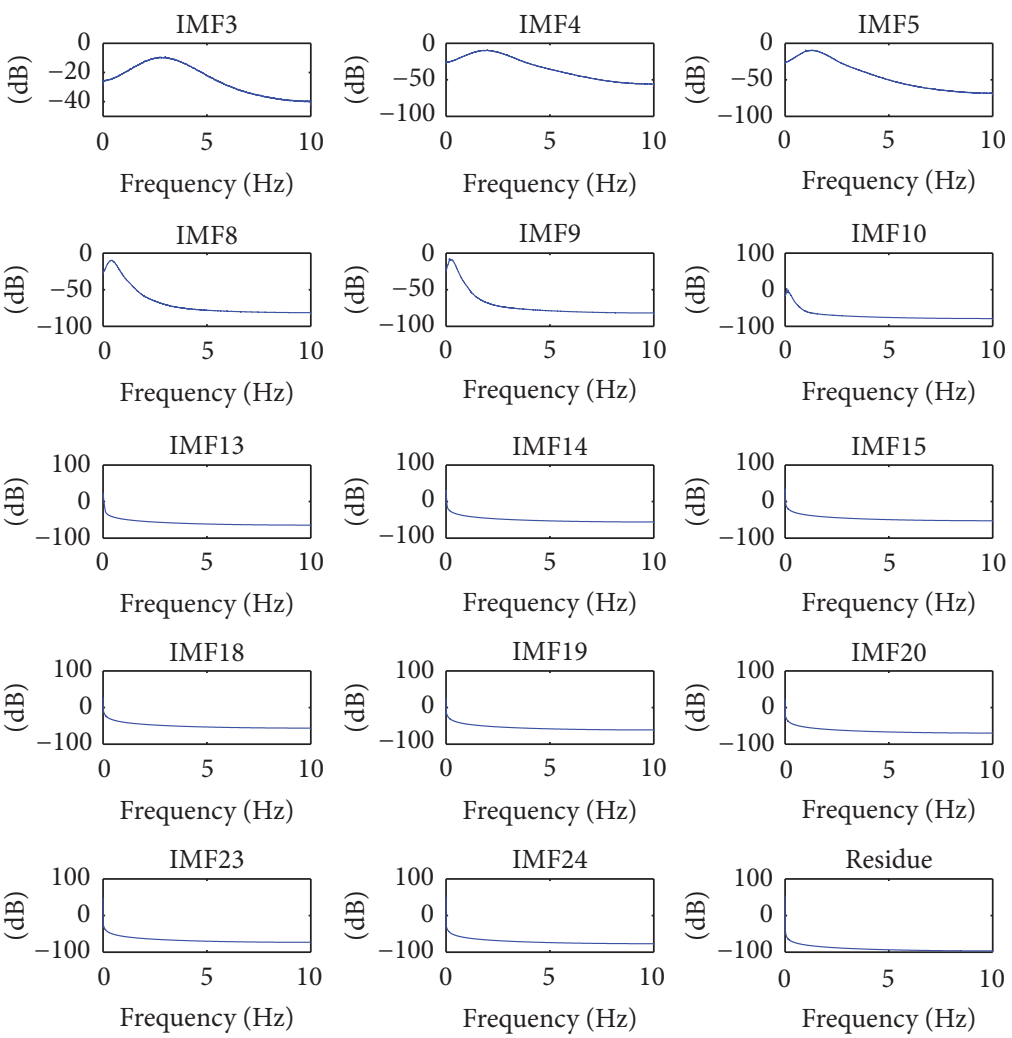

(b)
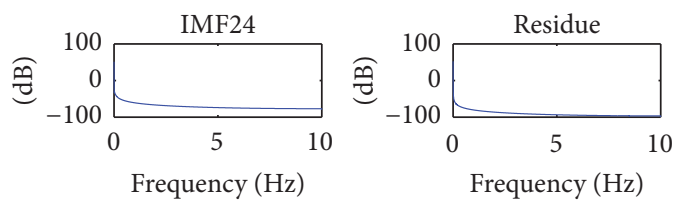

FIGURE 8: Decomposition results of the EMD method: (a) 24 IMF components and residues; (b) their PSDs. 


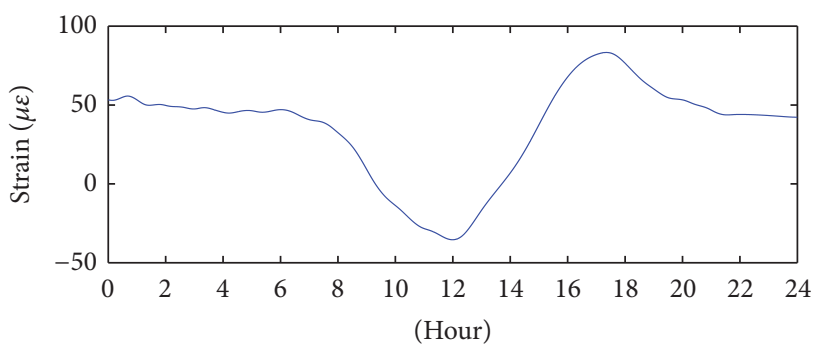

(a)

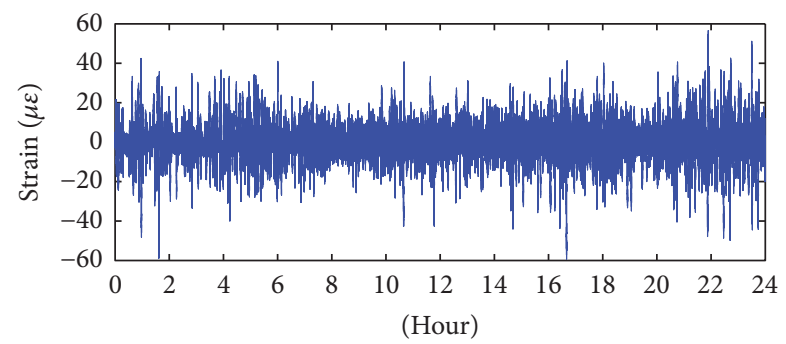

(b)

FIGURE 9: Extraction of the temperature-induced strain and dynamic strain from the raw signal: (a) temperature-induced strain; (b) dynamic strain.

TABLE 3: Stresses in the sensors on the upstream Yangzhong side.

\begin{tabular}{lccccc}
\hline Sensor & Height $(\mathrm{m})$ & Mean $(\mathrm{MPa})$ & SD $(\mathrm{MPa})$ & Measured max. $(\mathrm{MPa})$ & Expected max. $(\mathrm{MPa})$ \\
\hline B10 & \multicolumn{5}{c}{ Compression } \\
B9 & 13.915 & 1.2389 & 0.15798 & 1.5998 & 1.71284 \\
B5 & 53.657 & 4.1594 & 2.4424 & 13.4536 & 11.4866 \\
B4 & 66.5 & 4.70454 & 2.37432 & 13.03164 & 11.8275 \\
B3 & 79 & 4.1364 & 2.4142 & 14.0708 & 11.3792 \\
B2 & 135.46 & 4.4635 & 2.0539 & 11.3083 & 10.6253 \\
\hline & 159.46 & 2.0306 & 0.79832 & 5.29142 & 4.42556 \\
\hline B10 & & Tension & & -1.7214 \\
B9 & 13.915 & -1.2531 & 0.15612 & -1.7778 & -12.3299 \\
B5 & 53.657 & -4.3197 & 2.67 & -13.7625 & -13.1245 \\
B4 & 66.5 & -4.8447 & 2.7599 & -14.8101 & -12.6039 \\
B3 & 79 & -4.3147 & 2.7631 & -11.86 & -11.687 \\
B2 & 135.46 & -4.5421 & 2.3816 & -4.9527 & -4.6051 \\
\hline
\end{tabular}

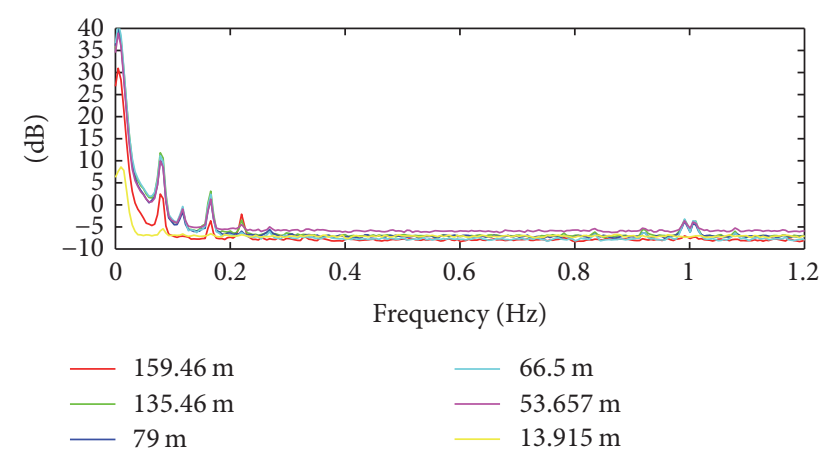

FIGURE 10: Frequency response from the dynamic strains at different heights.

and $159.46 \mathrm{~m}$, respectively. The highest mean tension is $-4.8447 \mathrm{MPa}$ at a height of $66.5 \mathrm{~m}$, which is 3.87 times and 2.33 times higher than those at heights of $13.915 \mathrm{~m}$ and $159.46 \mathrm{~m}$, respectively. The stress distribution with the height is shown in Figure 15.

Considering the structure's safety, the parts that bear considerable loads should use high strength steel. According to the results, the stresses at heights of $53.657 \mathrm{~m}$ to $135.46 \mathrm{~m}$ are higher than those at other heights, which indicates that

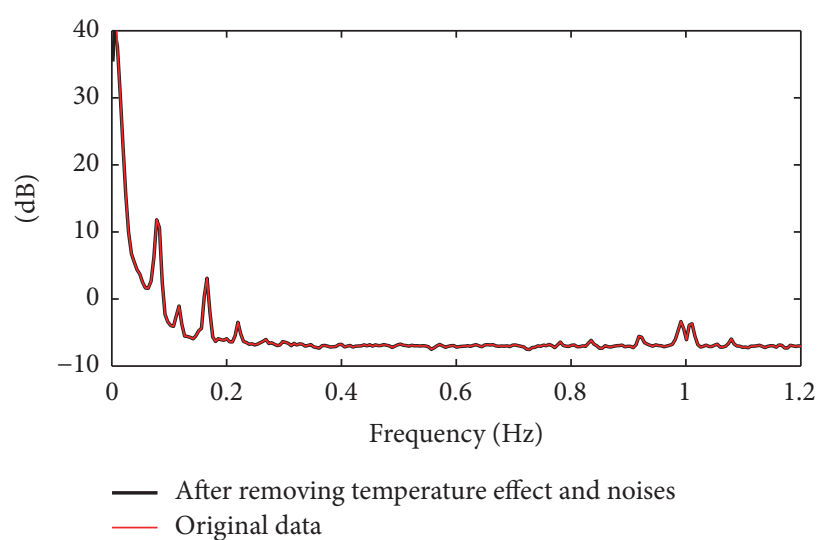

FIGURE 11: Frequency responses from both the original signal and the dynamic strain at a height of $135.46 \mathrm{~m}$.

high strength steel, such as Q420QD, should be used in the parts at these heights. This conclusion was also shown in the bridge design stage by Hua et al. [21], in which the stresses at heights of $51.9 \mathrm{~m}$ to $122 \mathrm{~m}$ were found to be significantly higher than the other stresses. This result also indicates that more sensors should be deployed in the stress concentration area to accurately assess the structural condition. 
TABLE 4: Stresses in the sensors on the upstream Yangzhong side.

\begin{tabular}{lccccc}
\hline Sensor & Height $(\mathrm{m})$ & Mean $(\mathrm{MPa})$ & SD $(\mathrm{MPa})$ & Measured max. $(\mathrm{MPa})$ & Expected max. $(\mathrm{MPa})$ \\
\hline B6 & & Compression & \\
B1 & 58.825 & 1.3182 & 0.15707 & 1.6862 & 1.78941 \\
\hline \multicolumn{7}{c}{188.593} & 1.2517 & 0.13864 & 1.6248 & 1.66762 \\
\hline B6 & 58.825 & -1.3438 & 0.17267 & -1.9645 & -1.8618 \\
B1 & 188.593 & -1.2968 & 0.17869 & -1.6799 & -1.8328 \\
\hline
\end{tabular}

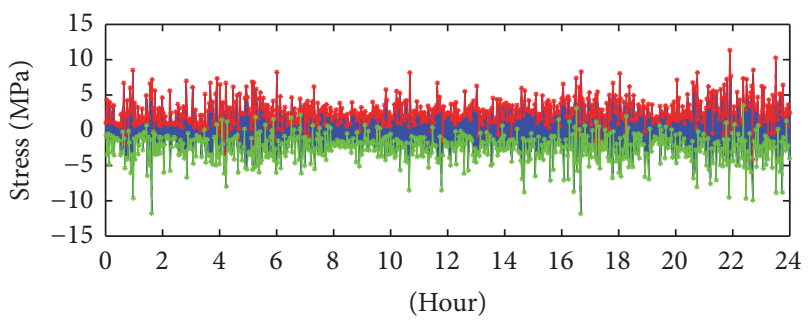

FIGURE 12: Extraction of the stress peaks and valleys.

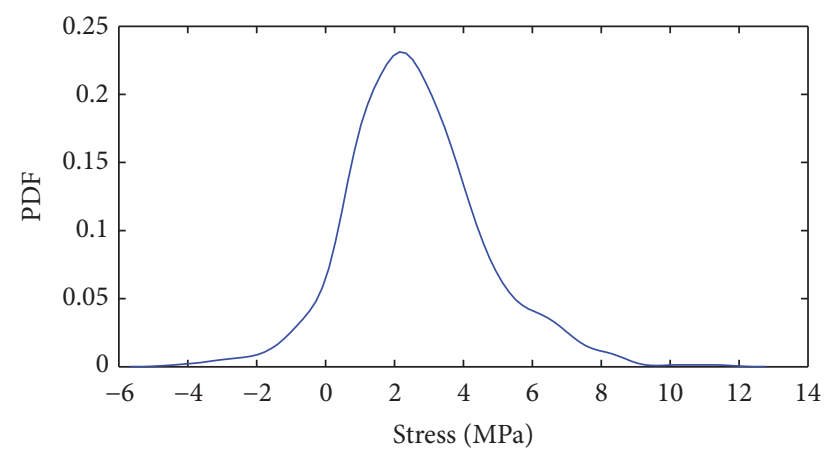

FIGURE 13: Distribution of the strain peaks of B3 on 2013-05-02.

Figure 15 shows that the stress distribution of the herringbone middle pylon exhibits high values in the middle and low values at both ends. Although the expected compressive and tensile stresses (absolute values) are slightly lower than the measured stresses at the same height, they show good agreement. These results indicate that the proposed model is suitable for simulating the distribution of peak stresses. Engineers can use the model to accurately predict the maximum stress in the middle tower for bridge maintenance. The results also demonstrate that the maximum compression and tension in the key segments of the middle tower exhibit considerable redundancy, which indicates that the middle pylon is in good condition.

5.2. Stress Analysis of the Beams. Table 4 lists the statistical parameters of the sensors installed in the upper and lower beams at heights of $188.593 \mathrm{~m}$ and $58.825 \mathrm{~m}$, respectively. The means and SDs are relatively small, as are the measured maximum stress and expected maximum stress. As a result, the stresses in the beams are not of critical concern in the structural condition assessment.

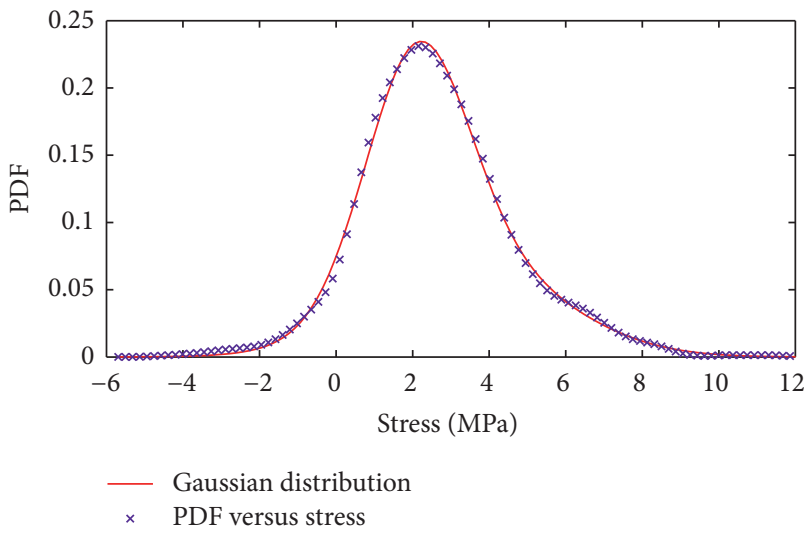

FIGURE 14: Distribution of the stress peaks and the Gaussian curve.

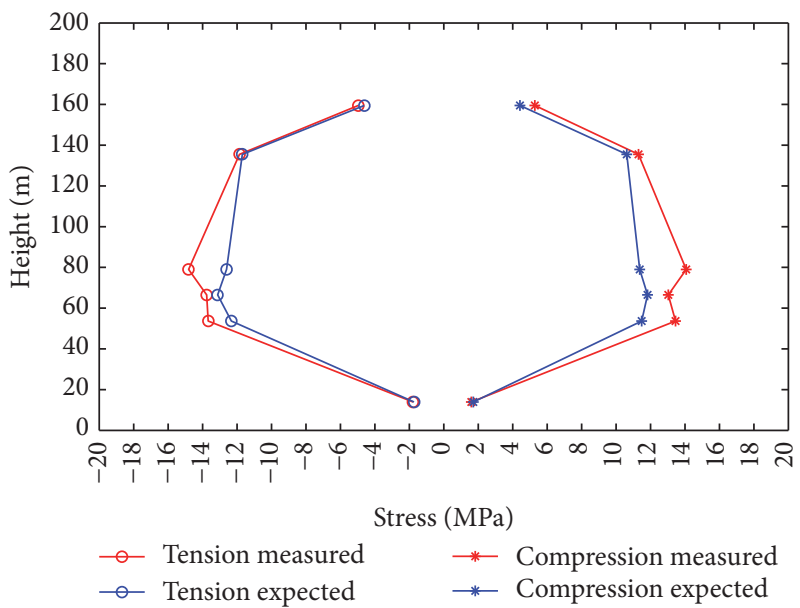

FIGURE 15: Variations of the measured and expected maximum compression and tensions with increasing tower height.

\section{Conclusions}

This paper presents a structural condition assessment of the herringbone steel middle tower of Taizhou Bridge. An EMD method was proposed to extract the dynamic strain of the steel middle pylon from the ESHMS installed in Taizhou Bridge. The structural characteristics, including frequency response and distribution of peak stresses, were analyzed for the structural condition assessment of the middle pylon. The results led to the following conclusions: 
(1) The proposed EMD method has a significant advantage over traditional parametric-based methods and waveletbased methods for complex original strain decomposition because of its adaptive and timescale-based decomposition properties. The method is applicable to nonlinear and nonstationary processes and is well suited for extracting the dynamic strain from the original signal.

(2) The temperature-induced strain and noise contribute little to the stress and have little influence on the frequency response of the middle pylon.

(3) The peaks of the dynamic stress in the middle pylon approximate a normal distribution. The R-squared value of the fit between the peaks and the normal distribution is determined to be 0.9981 .

(4) Assuming a normal distribution, the expected large stresses are found at heights of $53.657 \mathrm{~m}$ to $135.46 \mathrm{~m}$, and this trend agrees well with the measured results. Therefore, high strength steel should be used in this range of height, and the distribution model is suitable for predicting the maximum stress. In addition, the maximum compression and tension in the key segments of the middle tower exhibit considerable redundancy, which indicates that the middle pylon is in good condition.

\section{Conflicts of Interest}

The authors declare no conflicts of interest.

\section{Acknowledgments}

The authors acknowledge the Jiangsu Yangtze River Highway Bridge Construction Headquarters and Jiangsu Provincial Communications Planning and Design Institute Co., Ltd., for their support throughout the study. This work was supported by the Scientific and Technical Supporting Programs of Jiangsu Province, China (BE2011169).

\section{References}

[1] J. L. Brown, "Landmarks in American civil engineering history: golden gate bridge," Civil Engineering, vol. 72, no. 11, pp. 132-133, 2002.

[2] S. Adanur, M. Günaydin, A. C. Altunişik, and B. Sevim, "Construction stage analysis of Humber Suspension Bridge," Applied Mathematical Modelling, vol. 36, no. 11, pp. 5492-5505, 2012.

[3] Y. L. Xu, J. M. Ko, and Z. Yu, "Modal analysis of towercable system of Tsing Ma long suspension bridge," Engineering Structures, vol. 19, no. 10, pp. 857-867, 1997.

[4] H. Petroski, "Akashi kaikyo bridge," American Scientist, vol. 97, no. 3, pp. 192-196, 2009.

[5] J. B. Frandsen, "Simultaneous pressures and accelerations measured full-scale on the Great Belt East suspension bridge," Journal of Wind Engineering and Industrial Aerodynamics, vol. 89, no. 1, pp. 95-129, 2001.

[6] N. Apaydin and M. Erdik, "Structural vibration monitoring system for the bosporus suspension bridges," in Strong Motion Instrumentation for Civil Engineering Structures, pp. 342-367, Springer Netherlands, Dordrecht, Netherlands, 2001.
[7] D. Yang, D. Youliang, and L. Aiqun, "Structural condition assessment of long-span suspension bridges using long-term monitoring data," Earthquake Engineering and Engineering Vibration, vol. 9, no. 1, pp. 123-131, 2010.

[8] H. F. Zhou, Y. Q. Ni, and J. M. Ko, "Structural health monitoring of the Jiangyin Bridge: system upgrade and data analysis," Smart Structures and Systems, vol. 11, no. 6, pp. 637-662, 2013.

[9] K. Y. Wong, "Instrumentation and health monitoring of cablesupported bridges," Structural Control and Health Monitoring, vol. 11, no. 2, pp. 91-124, 2004.

[10] F. N. Catbas, M. Susoy, and D. M. Frangopol, "Structural health monitoring and reliability estimation: long span truss bridge application with environmental monitoring data," Engineering Structures, vol. 30, no. 9, pp. 2347-2359, 2008.

[11] Y. Q. Ni, H. W. Xia, K. Y. Wong, and J. M. Ko, "In-service condition assessment of bridge deck using long-term monitoring data of strain response," Journal of Bridge Engineering, vol. 17, no. 6, pp. 876-885, 2012.

[12] Y.-Q. Ni and Y.-X. Xia, "Strain-based condition assessment of a suspension bridge instrumented with structural health monitoring system," International Journal of Structural Stability and Dynamics, vol. 16, no. 4, Article ID 1640027, 2016.

[13] G.-X. Wang, Y.-L. Ding, P. Sun, L.-L. Wu, and Q. Yue, "Assessing static performance of the dashengguan yangtze bridge by monitoring the correlation between temperature field and its static strains," Mathematical Problems in Engineering, vol. 2015, Article ID 946907, 12 pages, 2015.

[14] Y. Deng, Y. Liu, D.-M. Feng, and A.-Q. Li, "Investigation of fatigue performance of welded details in long-span steel bridges using long-term monitoring strain data," Structural Control and Health Monitoring, vol. 22, no. 11, pp. 1343-1358, 2015.

[15] J. Li, J. Yan, T. Peng, and L. Han, "Shake table studies of seismic structural systems of a Taizhou Changjiang highway bridge model," Journal of Bridge Engineering, vol. 20, no. 3, Article ID 04014065, 2015.

[16] H. Wang, T. Tao, R. Zhou, X. Hua, and A. Kareem, "Parameter sensitivity study on flutter stability of a long-span triple-tower suspension bridge," Journal of Wind Engineering and Industrial Aerodynamics, vol. 128, pp. 12-21, 2014.

[17] H. W. Xia, SHM-based condition assessment of in-service bridge structures using strain measurement [Ph.D. thesis], Department of Civil and Environmental Engineering, The Hong Kong Polytechnic University, Hung Hom, Hong Kong, 2012.

[18] N. E. Huang, Z. Shen, S. R. Long et al., "The empirical mode decomposition and the Hilbert spectrum for nonlinear and non-stationary time series analysis," The Royal Society of London. Proceedings. Series A. Mathematical, Physical and Engineering Sciences, vol. 454, no. 1971, pp. 903-995, 1998.

[19] H. Wang, K. Zou, A. Li et al., "Parameter effects on the dynamic characteristics of a super-long-span triple-tower suspension bridge," Journal of Zhejiang University-Science A (Applied Physics \& Engineering), vol. 11, no. 5, pp. 305-316, 2010.

[20] Y. L. Deng, T. B. Peng, J. Z. Li, and L. Ji, "Study on dynamic characteristic and aseismic performance of a long-span tripletower suspension bridge," Journal of Vibration and Shock, vol. 27, no. 9, pp. 105-110, 2008.

[21] X. Hua, X. D. Zheng, Y. F. Zhou et al., "Design of middle steel tower on triple-tower suspension bridge of Taizhou Yangtze river bridge," Highway, vol. 7, pp. 68-74, 2009. 


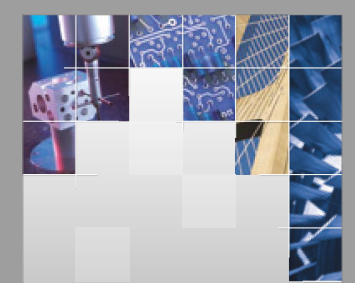

\section{Enfincering}
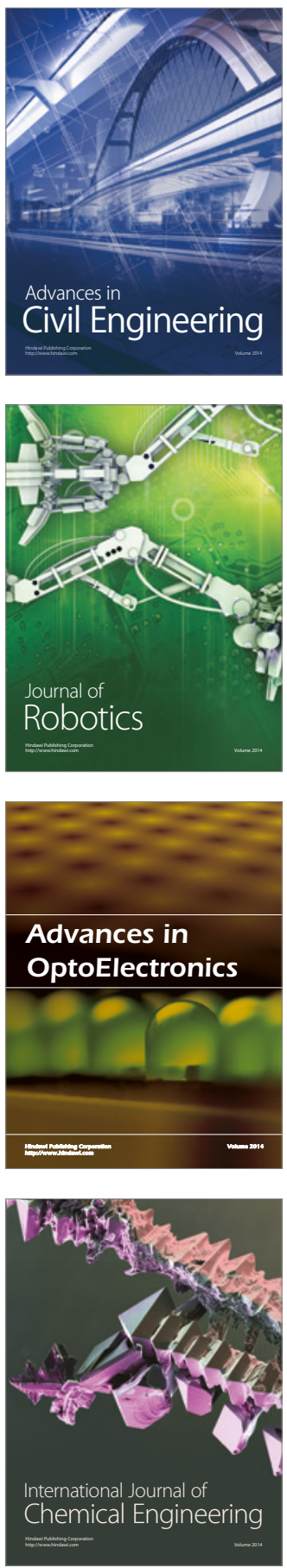

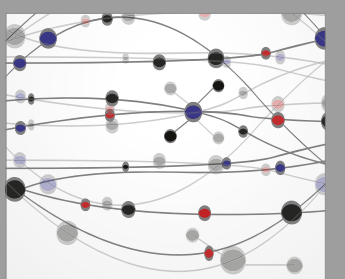

The Scientific World Journal

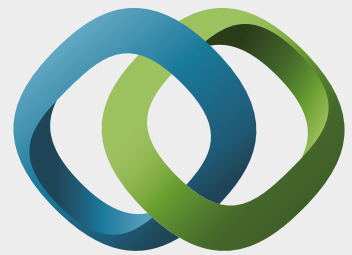

\section{Hindawi}

Submit your manuscripts at

https://www.hindawi.com
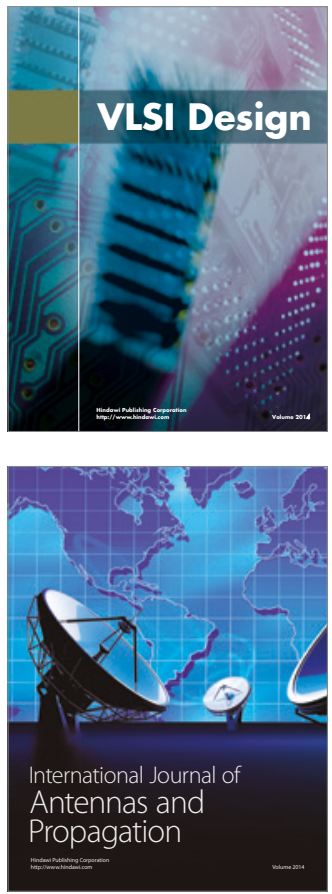

\section{Rotating}

Machinery
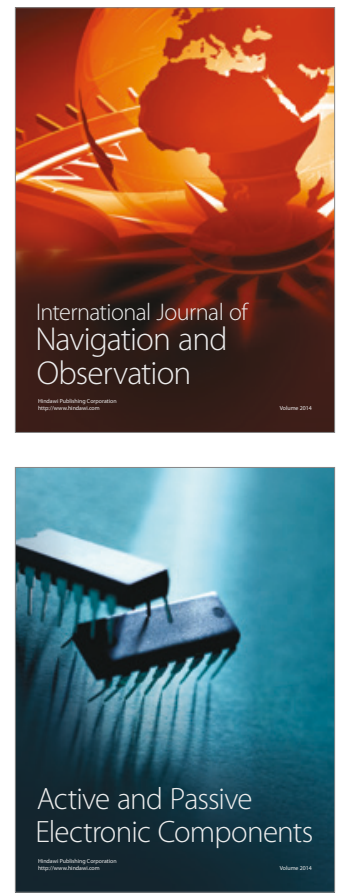
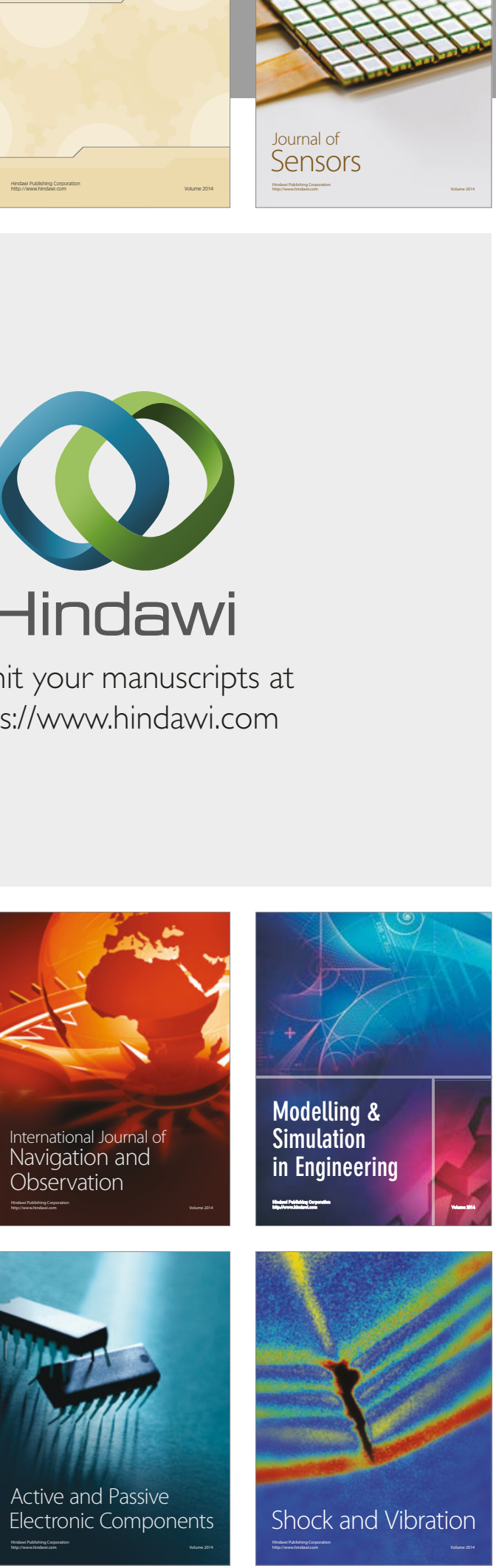
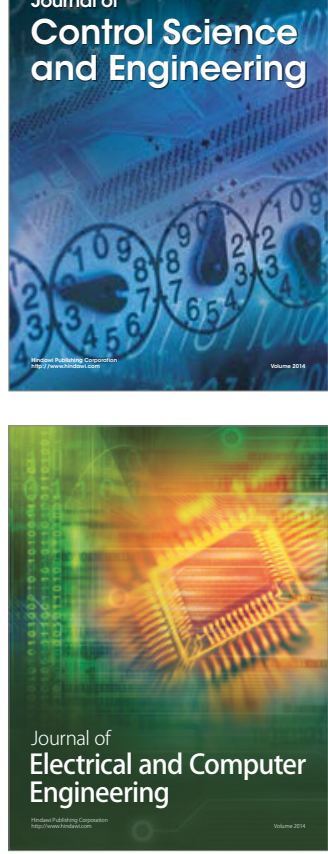

Distributed

Journal of

Control Science

and Engineering
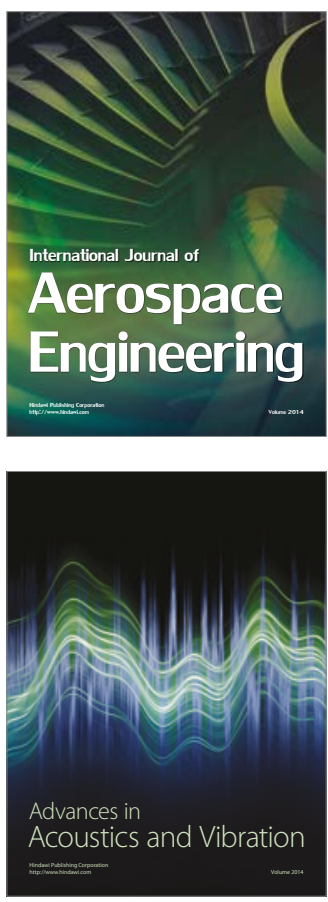

Sensor Networks 